\title{
OJO SECO Y CALIDAD DE VIDA
}

\author{
DRY EYE AND QUALITY OF LIFE
}

\author{
GARCÍA-CATALÁN MR ${ }^{1}$, JEREZ-OLIVERA E ${ }^{1}$, BENÍTEZ-DEL-CASTILLO-SÁNCHEZ JM²
}

\section{RESUMEN}

Objetivos: Evaluar la relación entre la severidad de ojo seco y la calidad de vida.

Material y métodos: 19 pacientes diagnosticados de ojo seco y 21 sin ojo seco fueron explorados con lámpara de hendidura y se les realizó el test de Schirmer, para evaluar el grado de severidad de ojo seco. La calidad de vida fue valorada con los cuestionarios OSDI (Ocular Surface Disease Index) and VFQ-25 (Visual Function Questionnaire-25).

Resultados: La puntuación total del OSDI era significativamente mayor en los pacientes con ojo seco, mientras que el BUT y la puntuación total del VFQ-25 era significativamente menor que en los sanos. En los casos, se encontraron las siguientes correlaciones estadísticamente significativas $(\mathrm{p}<0,05)$ : Puntuación total del OSDI con la puntuación total del VFQ-25 ( $\mathrm{r}=-0,62)$, BUT con la tinción corneal $(r=-0,50)$ y con el Schirmer $(r=0,66)$, BUT con la puntuación total del OSDI, los síntomas y los desencadenantes del OSDI ( $\mathrm{r}=-0,56,-0,56,-0,60)$; tinción corneal con la puntuación total del OSDI y los síntomas del OSDI ( $\mathrm{r}=0,55,0,54)$; BUT con la puntuación total del VFQ-25, la ausencia de dolor ocular, la función mental y la función laboral del VFQ-25 ( $\mathrm{r}=0,56,0,51,0,63,0,56)$; la tinción corneal con la puntuación total del VFQ-25, la ausencia de dolor ocular y la visión cercana del VFQ-25 ( $\mathrm{r}=-$ $0,57,-0,49,-0,62$ ).

\begin{abstract}
Purpose: To evaluate the relation between dry eye severity and quality of life.

Methods: 40 participants (19 with dry eye and 21 normal controls) underwent slit-lamp examination and Schirmer test to evaluate dry eye severity. Quality of life was evaluated with OSDI (Ocular Surface Disease Index) and VFQ-25 (Visual Function Questionnaire-25).

Results: The OSDI total score was significantly greater in patients suffering dry eye. However, BUT (break-up time) and VFQ-25 total score were significantly lower than in control subjects.

In dry eye patients several statistically significant correlations $(\mathrm{p}<0.05)$ were found:

OSDI with VFQ-25 total score ( $\mathrm{r}=-0.62)$, BUT with corneal staining $(\mathrm{r}=-0.50)$ and Schirmer (0.66), BUT with OSDI total score, OSDI-symptoms and OSDI-triggers $(\mathrm{r}=-0.56,-0.56,-0.60)$; corneal staining with OSDI total score and OSDI-symptoms $(\mathrm{r}=0.55,0.54)$, BUT with VFQ-25 total score, VFQ-25 ocular pain, mental function and role function. ( $\mathrm{r}=0.56,0.51,0.63,0.56)$; corneal staining with VFQ-25 total score, VFQ-25 ocular pain and near vision ( $\mathrm{r}=-0.57,-0.49,-0.62)$.

Conclusion: Quality of life is decreased in patients with dry eye. OSDI and VFQ-25 questionnaires are valid instruments for measuring the impact of dry eye disease. BUT and corneal staining provide an
\end{abstract}

\footnotetext{
Recibido: 21/7/08. Aceptado: 8/9/09.

Unidad de Superficie e Inflamación Ocular del Hospital Clínico San Carlos. Madrid. España.

${ }_{1}^{1}$ Licenciado en Medicina.

2 Doctor en Medicina.

Correspondencia:

María del Rocío García Catalán

Servicio de Oftalmología. USIO. Hospital Clínico San Carlos.

C/. Profesor Martín Lagos, s/n

28040 Madrid

España

E-mail: mariadelrociogarciacatalan@gmail.com
} 
Conclusión: Los pacientes con ojo seco tienen una peor de calidad de vida. Tanto el OSDI como el VFQ-25 son métodos adecuados de evaluación. El BUT y la tinción corneal proporcionan una medida indirecta de la calidad de vida.

Palabras clave: Ojo seco, OSDI, VFQ-25, BUT, tinción corneal. indirect measurement of quality of life (Arch Soc Esp Oftalmol 2009; 84: 451-458).

Key words: Dry eye, OSDI, VFQ-25, break-up time, corneal staining.

\section{INTRODUCCIÓN}

Según el DEWS 2007 (International Dry Eye WorkShop) podemos definir el ojo seco como una enfermedad multifactorial que afecta a la lágrima y a la superficie ocular, que resulta en síntomas de disconfort y alteración visual. Todo esto va acompañado de un aumento en la osmolaridad de la lágrima y de una inflamación de la superficie ocular.

El impacto del ojo seco sobre la calidad de vida viene determinado por: 1 . Dolor y síntomas irritativos, 2. Impacto sobre la función visual, 3. Efecto sobre la salud general y el bienestar. La necesidad de instilación frecuente de gotas lubricantes puede afectar a la interacción social y laboral (1).

Existen varios métodos válidos para valorar el efecto del ojo seco sobre la función visual y la calidad de vida. Alguno de ellos no son específicos como el SF-36 (Medical Outcome Study Short Form-36). Existen, sin embargo, cuestionarios específicos relacionados con la visión como el NEIVFQ (NEI-Visual Function Questionnaire), el OSDI (Ocular Surface Disease Index) o el IDEEL (Dry Eye on Everyday Life).

El OSDI (Ocular Surface Disease Index) es un cuestionario específico que incluye tres subescalas, referidas todas ellas a la última semana: 1. Disconfort ocular: hace referencia a síntomas como sensación de cuerpo extraño o dolor ocular; 2. Funcionalidad: mide la limitación para llevar a cabo tareas cotidianas como leer o usar el ordenador y 3. Factores ambientales: determina el impacto de los mismos en pacientes con ojo seco. Como posibles respuestas nos encontramos con: siempre, casi siempre, la mitad del tiempo, algunas veces y nunca. La puntuación final oscila en una escala de 0-100 donde la menor puntuación implica menos problemas y síntomas.

Por su parte, el VFQ-25 (Visual Function Questionnaire) es un cuestionario genérico para determinar el impacto de las patologías oculares en la cali- dad de vida. Está dividido en diferentes secciones que hacen referencia a: visión global (1), visión cercana (3), visión lejana (3), limitación en la función social (2), limitaciones en la función laboral (2), dependencia (3), salud mental (4), conducción (3), limitación en la visión periférica (1) y cromática (1) y dolor ocular (1). Como resultados obtenemos igualmente una escala con valores de 0-100 donde, en este caso, la menor puntuación supone una mayor sintomatología.

Con este estudio pretendemos establecer el grado de relación existente entre la severidad de ojo seco y la calidad de vida de los pacientes, haciendo uso para ello de dichos cuestionarios.

\section{SUJETOS, MATERIAL Y MÉTODOS}

Se reclutaron cuarenta sujetos, de los cuales, el grupo estudio estaba compuesto por 19 pacientes consecutivos diagnosticados de ojo seco en la consulta especializada de superficie ocular de nuestro hospital y el grupo control por 21 sanos que acudieron a la consulta general de oftalmología. Los controles fueron emparejados en función de la edad $( \pm 5$ años) y no podían tener una enfermedad sistémica asociada al ojo seco. Se excluyó a los pacientes con cirugía oftalmológica previa, alergia, infección ocular y aquellos con enfermedad sistémica no controlada que afectase a la vida diaria.

A todos los pacientes de les realizó una historia clínica y exploración con lámpara de hendidura. Se llevó a cabo tinción con fluoresceína $2 \%(0,05 \mathrm{ml})$ (Colircusi Fluoresceína, Alcon, El Masnou, Spain) para valorar el estado corneal y conjuntival, el tiempo de rotura de la lágrima (BUT) y el test de aclaramiento, así como tinción con rosa de Bengala. Se comprobó la posible disfunción de las glándulas de Meibomio y se practicó el test de Schirmer con anestésico para conocer la secreción lagrimal. 
La calidad de vida fue evaluada con ayuda de dos test específicos creados para este fin (OSDI y VFQ25). Todos los pacientes firmaron previamente un consentimiento informado. El análisis de los resultados fue realizado con el programa estadístico SPSS 10.0. Un valor de $\mathrm{p}<0,05$ fue considerado como significativo.

Tabla I. Diferencias entre casos y controles

\begin{tabular}{|c|c|c|}
\hline & Casos & Controles \\
\hline Edad media & $63,17 \mathrm{DE} 12,55$ & $63 \mathrm{DE} 11,81 \mathrm{p}>0,05$ \\
\hline \multicolumn{3}{|l|}{ Colirios } \\
\hline 0 & $0,00 \%$ & $95 \%$ \\
\hline 1 & $57,90 \%$ & $5 \%$ \\
\hline 2 & $26,30 \%$ & \\
\hline 3 & $15,80 \%$ & \\
\hline Higiene palpebral & $31,60 \%$ & $0 \%$ \\
\hline \multicolumn{3}{|c|}{ Hiperemia conjuntival } \\
\hline nada & $63,20 \%$ & $100 \%$ \\
\hline leve & $31,60 \%$ & \\
\hline moderado & $5,30 \%$ & \\
\hline \multicolumn{3}{|l|}{ Tinción conjuntival } \\
\hline nada & $52,60 \%$ & $86 \%$ \\
\hline leve & $31,60 \%$ & $14 \%$ \\
\hline moderado & $10,50 \%$ & \\
\hline grave & $5,20 \%$ & \\
\hline \multicolumn{3}{|l|}{ Tinción corneal } \\
\hline nada & $57,90 \%$ & $57,10 \%$ \\
\hline inferior & $15,80 \%$ & $33,30 \%$ \\
\hline central & $21,10 \%$ & $9,50 \%$ \\
\hline total & $5,30 \%$ & $0 \%$ \\
\hline \multicolumn{3}{|l|}{ DGM } \\
\hline nada & $10,50 \%$ & $76 \%$ \\
\hline leve & $63,20 \%$ & $19 \%$ \\
\hline moderado & $26,30 \%$ & $5 \%$ \\
\hline BUT & 4,73 DE 2,96 & 7,9 DE $3,12 p=0,003$ \\
\hline$>10 \mathrm{seg}$ & $10,50 \%$ & $38 \%$ \\
\hline Schirmer & 7,16 DE 1,7 & $12,14 \mathrm{DE} 1,5$ \\
\hline Aclaramiento & 0,26 DE 0,28 & 0,36 DE $0,27 \quad p>0,05$ \\
\hline OSDI & 40,18 DE 21,17 & 13,17 DE $9,96 \quad p<0,001$ \\
\hline VFQ-25 & $77,57 \mathrm{DE} 17,1$ & 90,13 DE $4,72 \mathrm{p}=0,006$ \\
\hline
\end{tabular}

Test de Mann-Whitney. DGM = Disfunción de las glándulas de meibomio. BUT $=$ Break-up time. OSDI $=$ Ocular Surface Disease Index . VFQ-25 = Visual Function Questionnaire-25. DE = desviación estándar.

\section{RESULTADOS}

La edad media de los casos fue de 63,17 DE 12,55 años y de 63 DE 11,81 años en los controles. El 89,5\% de los casos y el $85,7 \%$ de los controles eran mujeres, diferencias que no eran estadísticamente significativas.

Las diferencias entre casos y controles se exponen en la tabla I. Destacamos lo siguiente: En los casos, el BUT y la puntuación en el VFQ-25 era menor que en los controles mientras que la puntuación media del OSDI era superior. Estas diferencias resultaron estadísticamente significativas empleando el test de Mann-Whitney ( $\mathrm{p}=0,006)$.

\section{Asociación entre los parámetros de superficie ocular en los casos}

El BUT se correlacionaba con la tinción corneal y con el Schirmer de forma estadísticamente significativa. (Coeficiente de correlación de $-0,501$, $\mathrm{p}=0,029$ en el primer caso y de $0,663, \mathrm{p}=0,002$ en el segundo caso).

La tinción corneal se relacionaba de forma débil y no significativa con el Schirmer y la tinción conjuntival. (Coeficiente de Spearman de $-0,436 ; p=0,062$ y Spearman de 0,34; $p>0,05$ respectivamente).

\section{Asociación del OSDI con los parámetros de superficie ocular}

En el grupo de los casos, se encontró una asociación estadísticamente significativa entre la puntuación total del OSDI con el BUT y con la tinción corneal (tabla II). Con respecto al Schirmer, la asociación era débil y sin significación estadística.

En el grupo de los controles, solamente se encontró una correlación positiva de carácter moderado

Tabla II. Relación entre el OSDI (Ocular Surface Disease Index) u los parámetros de superficie ocular en los casos

\begin{tabular}{lcccrr}
\hline & & OSDI síntomas & OSDI función visual & OSDI desencadenantes & OSDI total \\
\hline Tinción corneal & Spearman & 0,539 & 0,239 & 0,430 & 0,075 \\
& $\mathrm{p}$ & 0,021 & 0,339 & $-0,188$ & 0,010 \\
BUT & Spearman & $-0,558$ & 0,456 & 0,009 & 0,013 \\
& $\mathrm{p}$ & 0,016 & 0,160 & $-0,516$ & $-0,273$ \\
Schirmer & Spearman & $-0,109$ & 0,525 & 0,028 & 0,258 \\
& $\mathrm{p}$ & 0,668 & & & \\
\hline \hline
\end{tabular}

BUT= Break-up time. OSDI = Ocular Surface Disease Index. 
entre la tinción corneal y la puntuación total del OSDI (fig. 1).

En los casos se estudió la posible relación entre los parámetros de superficie ocular con cada una de las subclasificaciones del cuestionario (OSDI-síntomas, OSDI-función visual y OSDI-desencadentantes (tabla II). Las mejores asociaciones obtenidas fueron para el BUT, que se correlacionaba de forma estadísticamente significativa con los síntomas y con los desencadenantes. Otras asociaciones estadísticamente significativas se encontraron entre la tinción corneal y los síntomas y entre el test de Schirmer y los desencadentes.

\section{Asociación del VFQ-25 con los parámetros de superficie ocular}

En los casos, de forma similar a lo encontrado con el OSDI, existía una asociación estadísticamente significativa entre la puntuación total del VFQ y el BUT, así como entre el VFQ-25 y la tinción corneal (figs. 2 y 3). Para el Schirmer, se encontró una débil correlación negativa que no alcanzó la significación estadística. En el grupo de los controles, no se encontraron asociaciones estadísticamente significativas.

Del mismo modo, estudiamos en los casos las relaciones entre los parámetros de superficie ocular

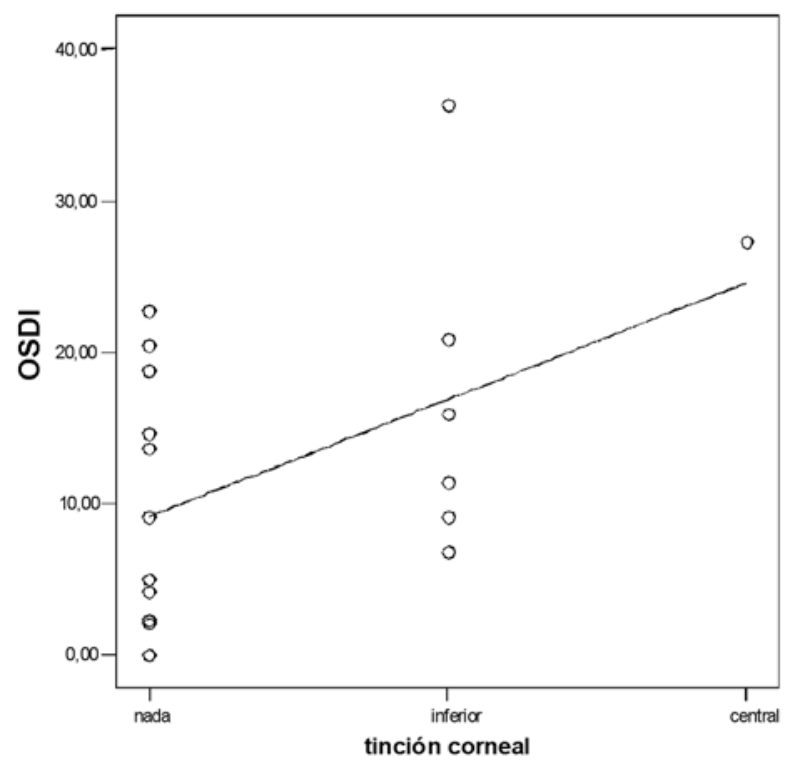

Fig. 1: Asociación entre el OSDI (Ocular Surface Disease Index) y la tinción corneal en los controles. Spearman $=0,5 ; p=0,02$.

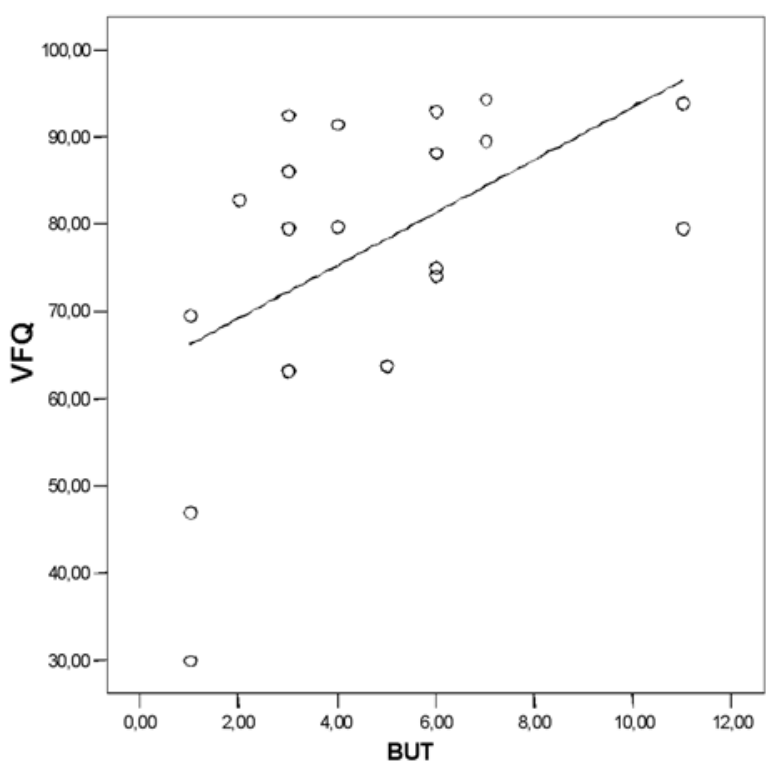

Fig. 2: Asociación entre el VFQ-25 (Visual Function Questionnaire-25) y el BUT en los casos. Spearman = 0,$56 ; p=0,011$.

con las distintas subescalas entre las que se divide el VFQ (tabla III). En cada una de ellas, una mayor puntuación en el VFQ-25 significa que el parámetro estudiado es menos patológico. Por ejemplo, en el caso de la subescala dolor ocular, a mayor pun-

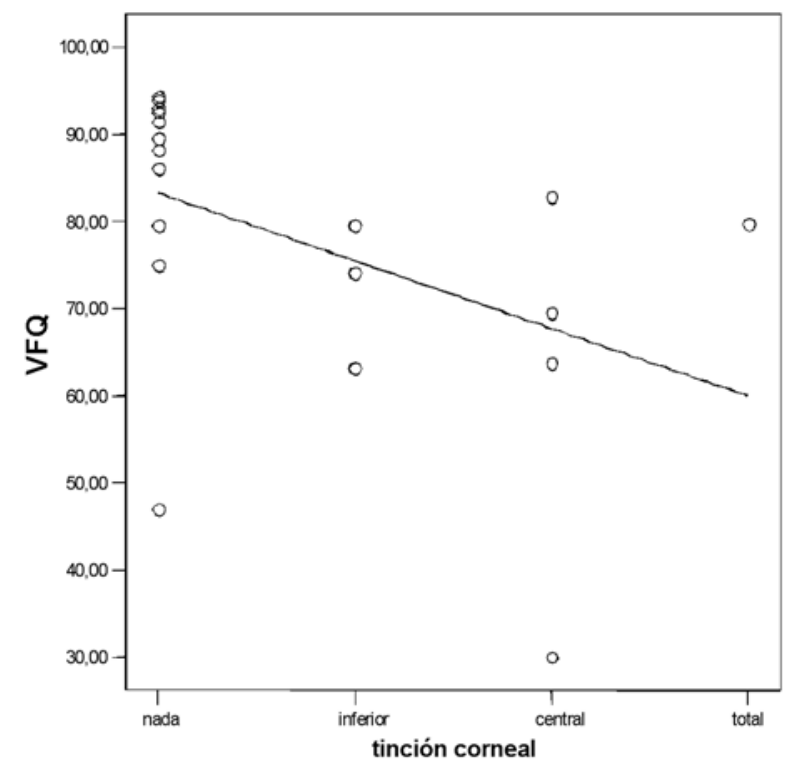

Fig. 3: Asociación entre el VFQ-25 (Visual Function Questionnaire-25) y la tinción corneal en los casos. Spearman $=-0,57 ; p=0,01$. 
Tabla III. Relación entre el VFQ-25 (Visual Function Questionnaire-25) y los parámetros de superficie ocular en los casos

\begin{tabular}{lccc}
\hline & Tinción corneal & BUT & Schirmer \\
\hline Visión global & $-0,27$ & $-0,11$ & $-0,18$ \\
Dolor ocular & $-0,49^{*}$ & $0,51^{*}$ & $0,53^{*}$ \\
Visión cercana & $-0,62^{*}$ & 0,44 & 0,27 \\
Visión lejana & $-0,36$ & 0,39 & 0,02 \\
Función social & $-0,37$ & 0,28 & 0,10 \\
Salud mental & $-0,21$ & $0,63^{*}$ & 0,46 \\
Función laboral & $-0,41$ & $0,56^{*}$ & 0,44 \\
Dependencia & $-0,14$ & 0,33 & 0,18 \\
VFQ total & $-0,57^{*}$ & $0,56^{*}$ & 0,31 \\
\hline
\end{tabular}

$* \mathrm{p}<0,05$. Test de Spearman. BUT $=$ Break-up time. VFQ $=$ Visual Function Questionnaire-25.

tuación en el VFQ, significa que el paciente percibe menos dolor

Las asociaciones más fuertes encontradas fueron entre el BUT y la función mental y entre la tinción corneal y la visión cercana. También hallamos correlación entre el dolor ocular con la tinción corneal, BUT y Schirmer y entre la función laboral y el BUT. Todas estas asociaciones fueron de carácter moderado y estadísticamente significativas (tabla III).

\section{Asociación entre el OSDI y el VFQ-25}

En los casos, se observó una correlación negativa, de carácter moderado y estadísticamente significativa entre las puntuaciones totales de ambos cuestionarios (Spearman de -0,62, p=0,004) (fig. 4). Sin embargo, en el grupo de los controles, la correlación fue de carácter muy débil y sin significación estadística (Spearman de $-0,07, \mathrm{p}>0,05$ ).

Debido a las múltiples correlaciones que se podrían establecer entre las subescalas de los dos cuestionarios, restringimos los resultados a aquellos en donde pensamos que podría existir una asociación. En concreto, buscamos en primer lugar una asociación entre el dolor ocular del VFQ y las tres subdivisiones del OSDI y en segundo lugar la relación entre la función visual del OSDI con los siguientes parámetros del VFQ: visión global, visión cercana y lejana, salud mental, dependencia, conducción, función social y laboral.

El parámetro dolor ocular del cuestionario VFQ25 se correlacionó de forma significativa con el parámetro desencadenantes del OSDI. Esta asociación fue negativa y de carácter moderado (Spearman de $-0,59, p=0,01$ ).

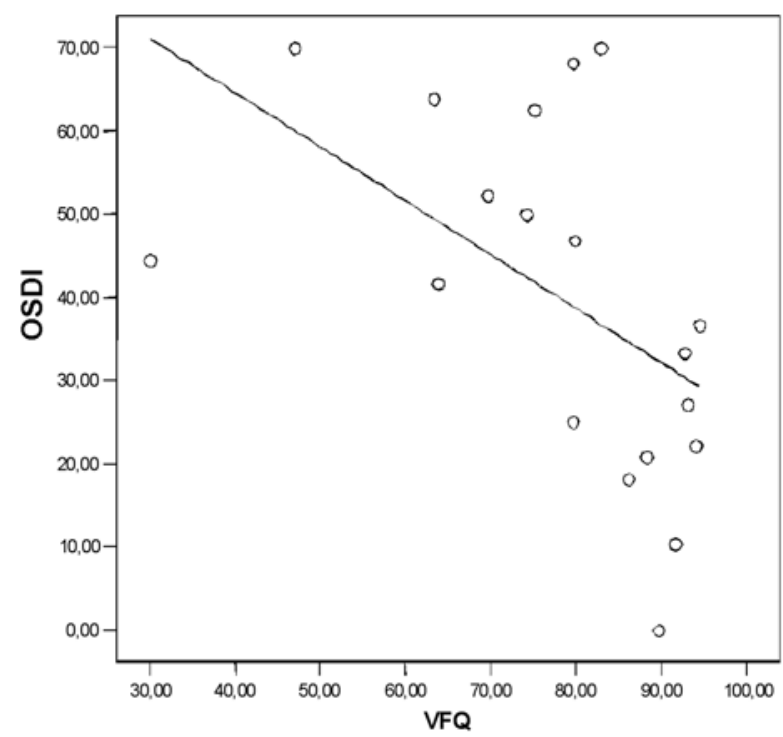

Fig. 4: Asociación entre el OSDI (Ocular Surface Disease Index) y el VFQ (Visual Function Questionnaire-25) en los casos Spearman $=-0,62 ; p=0,004$.

El ítem función visual del OSDI se correlacionó de forma significativa con la visión global, la visión cercana, la visión lejana y la dependencia del cuestionario VFQ-25. (Asociación negativa en todos los casos). Las asociaciones más fuertes encontradas fueron para la visión lejana y para la visión cercana (Spearman de $-0,8$ y de $-0,69$. respectivamente, $\mathrm{p}<0,01)$. Los parámetros visión global y dependencia presentaron una asociación de carácter moderado. (Coeficientes de $-0,49$ y $-0,55$ respectivamente; $\mathrm{p}=0,04$ y $\mathrm{p}=0,01)$.

\section{Asociación entre ambos cuestionarios con el número de colirios}

No se encontró una relación estadísticamente significativa entre el número de colirios y la puntuación total de los cuestionarios.

\section{DISCUSIÓN}

La puntuación media del OSDI en nuestros pacientes era superior a la registrada por Vitale (30) y Schiffman (21) (2,3). Dentro del ojo seco, los pacientes con síndrome de Sjögren padecen de una mayor intensidad y frecuencia de síntomas que aquellos con ojo seco no Sjögren (4). Todos los 
pacientes del estudio de Vitale sufrían de síndrome de Sjögren, mientras que en el estudio de Schiffman y en el nuestro, se engloba a pacientes con y sin síndrome de Sjögren, por lo que la puntuación del OSDI en nuestro trabajo debería ser menor que la recogida por Vitale. Sin embargo, el OSDI, no solo recoge los síntomas, sino que además tiene en cuenta la habilidad para llevar a cabo actividades de la vida diaria y la percepción de la propia salud.

Algunos estudios llegan a la conclusión de que el OSDI es un test que demuestra una alta sensibilidad y especificidad para distinguir entre sujetos normales y enfermos (3). En nuestro trabajo la puntuación del OSDI era significativamente mayor en los casos que en los controles.

El Schirmer medio en nuestros pacientes era mayor que el recogido por Vitale y $\operatorname{Ram}(4,8 \mathrm{~mm})$ $(2,5)$. Mientras que estos dos autores realizaron el test Schirmer sin anestésico, nosotros realizamos el Schirmer con anestésico. Esperábamos obtener una puntuación menor que en dichos estudios, ya que el Schirmer con anestesia mide solamente la secreción basal, a diferencia del test sin anestesia que expresa la secreción basal y refleja.

El Schirmer ha sido criticado por la variabilidad y por la poca reproducibilidad. Esta variabilidad es mayor cuando se emplea anestésico. Incluso anestesiando la córnea y la conjuntiva, la secreción lagrimal es llevada a cabo por estímulos sensoriales a través de los párpados y pestañas. Además hay que dejar tiempo entre la gota y el test para minimizar el lagrimeo reflejo producido por la sensación de escozor. Por otra parte, muchos pacientes con Schirmer bajos tienen pocos síntomas, mientras que otros con una moderada secreción lagrimal pueden desarrollar queratoconjuntivitis sicca grave (6).

El BUT medio de nuestros casos era similar al encontrado por Vitale y Ram (2,9 y 4 segundos respectivamente) $(2,5)$.

\section{Parámetros de superficie ocular}

En la literatura encontramos correlaciones estadísticamente significativas entre el BUT y la tinción corneal $(-0,439 ;-0,07)(4,7)$ entre el BUT y el Schirmer $(0,25) 7$ y entre el Schirmer y la tinción corneal $(-0,197 ;-0,13)(4,7)$.

Acorde con lo publicado, en nuestro estudio, existe asociaciones estadísticamente significativas entre el BUT y la tinción corneal y entre el BUT y el Schirmer. En el caso del Schirmer y la tinción corneal, no se alcanzaba la significación estadística.

A diferencia del trabajo de Begley, no hemos obtenido una correlación estadísticamente significativa entre la tinción conjuntival y la corneal, que puede ser debido a nuestro pequeño tamaño muestral. En nuestro estudio, la fuerza de la asociación era menor que en la de este autor $(r=0,35)(4)$.

\section{OSDI total-VFQ total}

En nuestro estudio hemos encontrado una correlación ente los cuestionarios OSDI y VFQ-25 similar a la descrita por Vitale (Coeficiente de -0,6) pero inferior a la descrita por Schiffman (coeficiente de correlación de -0,77) $(2,3)$.

\section{Colirios-OSDI}

Schiffman objetivó una correlación estadísticamente significativa entre el número de colirios y la puntuación total del OSDI (Coeficiente de correlación de 0,45) (3). En nuestro caso no se alcanzó la significación estadística.

\section{OSDI total-Tinción corneal, BUT, Schirmer}

Algunos estudios solamente han encontrado una débil correlación entre los síntomas y signos del ojo seco (2). Schein no encontró correlación entre el Schirmer y los síntomas, mientras que la tinción corneal sí se correlacionaba, aunque de forma débil, con los síntomas (8). Por el contrario, Lin observó que un Schirmer menor de $5 \mathrm{~mm}$ sí se relacionaba de forma significativa con una mayor frecuencia de síntomas, a diferencia de la tinción corneal. Lin piensa que la menor disfunción de las glándulas de Meibomio en la raza asiática en comparación con la raza blanca podría explicar esta diferencia (7).

En nuestro estudio encontramos correlaciones estadísticamente significativas para la tinción corneal y para el BUT, con una fuerza de asociación mayor que lo descrito en la literatura $(-0,29$ en el caso del BUT y 0,19 en la tinción corneal) $(2,3)$. Sin embargo, para el Schirmer, no se alcanzó la significación estadística, a diferencia de lo encontrado por Schiffman (3). 


\section{Puntuación total VFQ-25-Tinción corneal, BUT, Schirmer}

Vitale encontró una correlación entre la puntuación total y estos tres parámetros $(0,2,0,24$ y 0,19 respectivamente), aunque ninguna alcanzó significación estadística. En cambio, en nuestro estudio, la asociación entre la puntuación total con la tinción corneal y con el BUT sí eran estadísticamente significativas y además, la fuerza de la asociación era mayor (2). Con respecto al Schirmer tampoco observamos diferencias significativas.

Dado que el OSDI es un cuestionario más específico para el ojo seco que el VFQ, esperábamos encontrar mejores correlaciones entre los parámetros de superficie ocular y la puntuación total OSDI que con la del VFQ. Sin embargo, la fuerza de la asociación era similar para el BUT, pero ligeramente mayor para el VFQ en el caso de la tinción corneal.

\section{Subescalas OSDI-superficie ocular}

Vitale no encontró asociaciones estadísticamente significativas. La relación más fuerte observada era entre la función visual y el BUT (Spearman de $0,22)(2)$. En nuestro trabajo, los síntomas y los desencadenantes del OSDI sí se relacionaban de forma significativa con el BUT.

\section{Subescalas VFQ-superficie ocular}

En el estudio de Vitale, ninguna asociación resultó estadísticamente significativa y en general eran más débiles. Las asociaciones más fuertes que encontró fueron visión lejana-tinción corneal, visión cercana-BUT ( $\mathrm{r}=0,33$ y 0,32 respectivamente) (2). La relación entre la visión cercana y el BUT está en consonancia con lo encontrado frecuentemente en la práctica clínica, donde los pacientes con ojo seco refieren un empeoramiento de la presbicia, que mejora al pedirles que parpadeen con más frecuencia. Nosotros hemos encontrado una correlación estadísticamente significativa entre la visión cercana y la tinción corneal.

$\mathrm{Al}$ estudiar las subescalas de ambos cuestionarios con los parámetros de superficie ocular, las del OSDI presentaron coeficientes de correlación mejores que las del VFQ.

\section{Subescalas OSDI-subescalas VFQ}

Vitale encontró correlaciones estadísticamente significativas entre el dolor ocular del VFQ-25 con los síntomas oculares y la función visual del OSDI. (Spearman de 0,6 y 0,5 respectivamente) (2). En nuestro estudio no se observó significación estadística para estos dos parámetros, aunque sí se encontró para el ítem desencadenantes oculares del OSDI.

En cuanto a la función visual del OSDI, Vitale halló relaciones estadísticamente significativas con la visión general, dependencia, salud mental, función laboral, y conducción. ( $\mathrm{r}=0,6,0,61,0,64,0,42$ y 0,57$)(2)$. Nosotros hemos observado significación estadística para la visión general, dependencia, visión cercana y visión lejana.

En conclusión:

- Los pacientes con ojo seco tienen una peor calidad de vida que los individuos sanos.

- Tanto el OSDI como el VFQ-25 son medios adecuados para evaluar la calidad de vida en pacientes con ojo seco. (Buena correlación entre la puntuación total de los cuestionarios y entre subparámetros que valoran mismos aspectos de la calidad de vida). Aunque el VFQ-25 es de carácter menos específico para el ojo seco que el OSDI, la puntuación total del VFQ-25 se correlaciona mejor con los parámetros de superficie ocular que el OSDI. Sin embargo, si tenemos en cuenta las subescalas, las del OSDI se correlacionan mejor que las del VFQ25. Estas diferencias no parecen clínicamente relevantes, por lo que el OSDI, al ser más sencillo, es un cuestionario más útil.

- El BUT y la tinción corneal son herramientas útiles en el diagnóstico de pacientes con ojo seco y pueden proporcionar una medida indirecta de la calidad de vida.

- El Schirmer parece menos útil en cuanto a la estimación de la calidad de vida.

\section{BIBLIOGRAFÍA}

1. Lemp MA. The definition and classification of dry eye disease: Report of the definition and classification subcommittee of the international dry eye workshop (2007). Ocul Surf 2007; 5: 75-92.

2. Vitale S, Goodman LA, Reed GF. Comparison of the NEI$V F Q$ and OSDI questionnaires in patients with Sjögren's syndrome-related dry eye. Health Qual Life Outcomes 2004; 1: 44-54. 
3. Schiffman RM, Christianson MD, Jacobsen G, Hirsch JD, Reis BL. Reliability and validity of the ocular surface disease index. Arch Ophthalmol 2000; 118: 615-621.

4. Begley CG, Caffery B, Chalmers RL, Mitchell GL. Use of the dry eye questionnaire to measure symptons of ocular irritation in patients with aqueous tear deficient dry eye. Cornea 2002; 21: 664-670.

5. Ram J, Gupta A, Brar GS, Kaushik S, Gupta A. Outcomes of phacoemulsifiction in patients with dry eye. J Cataract Refract Surg 2002; 28: 1386-1389.

6. Macri A, Pflugfelder S. Correlation of the Schirmer 1 and fluorescein clearance tests with the severity of corneal epithelial and eyelid disease. Arch Ophthalmol 2000; 118: 1632-1638.

7. Lin PY, Cheng CY, Hsu WM, Tsai SY, Lin MW, Liu JH et al. Association between symptoms and signs of dry among an elderly chinese population in Taiwan. Invest Ophthalmol Vis Sci 2005; 46: 1593-1598.

8. Schein OD, Tielsch JM, Muñoz B, Bandeen-Roche K, West $S$. Relation between signs and symptoms of dry eye in the elderly. A population-based perspective. Ophthalmology 1997; 104: 1395-1401. 